\title{
Low-scale leptogenesis assisted by a real scalar singlet
}

\author{
Tommi Alanne, ${ }^{*}$ Thomas Hugle, ${ }^{\dagger}$ Moritz Platscher, ${ }^{\ddagger}$ \\ Max-Planck-Institut für Kernphysik, Saupfercheckweg 1, 69117 Heidelberg, Germany \\ and Kai Schmitz ${ }^{\S}$ \\ Dipartimento di Fisica e Astronomia, Università degli Studi di Padova, Via Marzolo 8, 35131 Padova, Italy \\ Istituto Nazionale di Fisica Nucleare (INFN), Sezione di Padova, Via Marzolo 8, 35131 Padova, Italy
}

\begin{abstract}
Standard thermal leptogenesis in the type-I seesaw model requires very heavy right-handed neutrinos (RHNs). This makes it hard to probe this scenario experimentally and results in large radiative corrections to the Higgs boson mass. In this paper, we demonstrate that the situation is considerably different in models that extend the Higgs sector by a real scalar singlet. Based on effective-theory arguments, the extra scalar is always allowed to couple to the heavy neutrinos via singlet Yukawa terms. This opens up new RHN decay channels leading to larger $C P$ violation as well as to a stronger departure from thermal equilibrium during leptogenesis. As a consequence, the baryon asymmetry can be generated for a lightest RHN mass as low as $500 \mathrm{GeV}$ and without the need for a highly degenerate RHN mass spectrum. In fact, the requirement of successful leptogenesis via the Higgs portal coupling singles out an interesting parameter region that can be probed in on-going and future experiments. We derive a semianalytical fit function for the final baryon asymmetry that allows for an efficient study of parameter space, thus enabling us to identify viable parameter regions. Our results are applicable to a wide range of models featuring an additional real scalar singlet.
\end{abstract}

\section{INTRODUCTION}

The origin of the baryon asymmetry of the Universe (BAU), typically quantified in terms of the cosmic baryonto-photon ratio $\eta_{B}^{\text {obs }} \simeq 6.1 \times 10^{-10}[1,2]$, is one of the greatest mysteries in fundamental physics. An attractive approach towards this problem consists in baryogenesis via leptogenesis [3] in the context of the type-I seesaw mechanism [4-8]. Leptogenesis is motivated by the fact that it links the generation of the BAU at high energies to the phenomenology of neutrino oscillations at low energies. Depending on further model assumptions, this connection allows one to derive testable predictions in the neutrino sector based on the requirement of successful leptogenesis (see Refs. [9-14] for a recent series of review articles).

The type-I seesaw model postulates the existence of at least two right-handed neutrinos (RHNs), $N_{i}$, that transform as complete singlets under the Standard Model (SM) gauge group and whose Majorana masses, $M_{i}$, violate lepton number by two units (see, e.g., Refs. [15-17] for minimal realizations of the type-I seesaw model). The $C P$-violating out-of-equilibrium decays of these neutrinos in the early Universe generate a primordial lepton asymmetry that is subsequently converted into a primordial baryon asymmetry by means of electroweak (EW)

\footnotetext{
* tommi.alanne@mpi-hd.mpg.de

† thomas.hugle@mpi-hd.mpg.de

‡ moritz.platscher@mpi-hd.mpg.de

$\S$ kai.schmitz@pd.infn.it
}

sphaleron processes [18]. However, in the standard scenario of thermal leptogenesis, the amount of $C P$ violation in RHN decays turns out to be suppressed by the tiny masses of the active SM neutrinos, which results in a strong lower bound on the RHN mass scale [19]. A simplified treatment, not taking into account the dynamics in flavor space, leads to the conclusion that the lightest RHN mass, $M_{1}$, must be at least of $\mathcal{O}\left(10^{9}\right) \mathrm{GeV}$ [20-22]. The inclusion of flavor effects, on the other hand, can lower this bound by up to three orders of magnitude, $M_{1} \gtrsim 10^{6} \mathrm{GeV}[23-26]$ - but certainly not to the extent that the RHN mass scale would approach the energy range that is probed by current or near-future colliders. The direct observation of heavy Majorana neutrinos in terrestrial experiments therefore seems to be out of reach in the standard scenario, at least in the foreseeable future. In addition to this phenomenological drawback, standard thermal leptogenesis is also plagued by a pressing theoretical issue: large radiative corrections that arise in the RHN sector at the one-loop level. Indeed, the large hierarchy between the RHN mass scale and the EW scale, $M_{i} \gg v_{\text {ew }} \simeq 246 \mathrm{GeV}$, threatens to destabilize the Higgs mass, which necessitates some severe fine-tuning of input parameters [27], as long as no other stabilization mechanism is at work. ${ }^{1}$

\footnotetext{
${ }^{1}$ Note, however, that the radiative corrections in the type-I seesaw model may also be used to explain the very origin of the SM Higgs potential. This is the basic idea behind the so-called "neutrino option" [28-31], which assumes that the classical SM Lagrangian satisfies scale-invariant boundary conditions at high energies.
} 
Together, these observations motivate efforts to seek alternative realizations of the leptogenesis paradigm that allow for the possibility of successful baryogenesis at a significantly lower RHN mass scale. Two popular approaches in this direction are (i) resonant leptogenesis [32, 33], which assumes a highly degenerate RHN mass spectrum, and (ii) leptogenesis via the Akhmedov-Rubakov-Smirnov mechanism of RHN oscillations [34] (see Refs. [35, 36] for very recent work based on these ideas). Alternatively, one may consider slight modifications of the type-I seesaw model that provide one with a larger parametric freedom in generating the BAU [37]. One such example is, e.g., leptogenesis in the scotogenic model of radiative neutrino masses [38]. This model promotes the type-I seesaw sector to a "dark sector", where the sterile neutrinos couple to a second, dark Higgs doublet, and in which all fields transform odd under a new discrete $\mathbb{Z}_{2}$ symmetry. As recently shown, the additional parameters present in this scenario allow one to lower the energy scale of leptogenesis down to RHN masses of $\mathcal{O}(10) \mathrm{TeV}$ [39]. For further studies of baryogenesis in the scotogenic model, see Refs. [40-44].

In this paper, we will study another opportunity for low-scale leptogenesis that builds upon the general typeI seesaw framework. As shown in Refs. [45, 46], this mechanism, while being more minimal than leptogenesis in the scotogenic model, allows for a significantly lower RHN mass scale and, as we will demonstrate, manages to successfully generate the BAU for RHN masses even below the $\mathrm{TeV}$ scale and still does not require a strong RHN mass degeneracy. While the scotogenic model assumes the existence of an extra scalar doublet, we will consider in this paper the mere addition of an extra real scalar singlet, $S$. The scalar sector thus represents the simplest conceivable extension of the SM Higgs sector. In the main part of our analysis, we will demonstrate how the presence of the scalar $S$ modifies some of the key quantities in the RHN sector and develop a numerical as well as analytical understanding of the ensuing leptogenesis scenario. This analysis will enable us to identify viable parameter regions that can be probed in on-going and future experiments.

The existence of an extra real scalar singlet is well motivated and frequently encountered in various more fundamental models. If the scalar $S$ is stable, it represents, e.g., a viable and minimal particle candidate for dark matter [47-50]. Alternatively, it is possible to identify $S$ with the inflaton field, which is responsible for a stage of cosmic inflation in the early Universe [51-54] (see also Ref. [55]). A third possibility, which has been intensively studied in the literature, is to associate the field $S$ with the dynamics of EW symmetry breaking, in particular, in such a way that the EW phase transition turns into a strong first-order phase transition [56-61]. Similarly, the field $S$ might correspond to a pseudo-Nambu-Goldstone boson (pNGB) in models that attribute the origin of the SM Higgs potential to the spontaneous breaking of an approximate global symmetry. In Ref. [62], it was shown that, coupling such a pNGB singlet to RHNs, it is possible to connect low-scale leptogenesis to EW symmetry breaking and Goldstone-Higgs models. From a theoretical perspective, the singlet $S$ is motivated by the fact that it allows one to ensure absolute stability of the EW vacuum, as opposed to the metastability of the EW vacuum in the pure standard model [63, 64]. Finally, the extension of the SM by a real scalar singlet represents an important experimental benchmark scenario for new-physics searches at present and future colliders [65-71].

We stress that, despite this broad spectrum of possible applications, the physical origin of the field $S$ is, in fact, irrelevant for our purposes. As we will see in the course of our analysis, leptogenesis will not require us to specify an ultraviolet (UV) completion of our model. Instead, it will suffice to regard the field $S$ as part of a low-energy effective description that descends from some unknown dynamics at high energies. In this sense, our analysis will follow a bottom-up approach that promises to be compatible with a large range of UV-complete models.

Our crucial observation is that the singlet $S$ is, a priori, always allowed to couple to the heavy Majorana neutrinos in the seesaw sector, $\mathcal{L} \supset S N N$. This operator is renormalizable and trivially invariant under all SM gauge symmetries. It explicitly breaks the accidental global lepton-number symmetry of the SM, but the same is also true for the large RHN Majorana masses in the seesaw Lagrangian. We therefore argue that the singlet-neutrino Yukawa coupling $S N N$ is likely to be present in any lowenergy effective theory that contains a real scalar singlet and a set of sterile neutrinos. This is a characteristic and fascinating consequence of combining two popular SM extensions: the real-scalar-singlet extension of the Higgs sector on the one hand and the type-I seesaw extension of the neutrino sector on the other hand. Note that, apart from Higgs portal couplings in the potential, other renormalizable couplings between the singlet and SM degrees of freedom (DOFs) are forbidden by gauge invariance.

The operator $S N N$ has important consequences for leptogenesis. In this paper, we will study its impact assuming a minimal singlet sector that consists of only two heavy neutrinos $N_{1,2}$ and one real scalar $S$. This restriction will help us simplify our analysis, but at the same time still manage to capture all relevant effects. At tree level, the presence of a light scalar $S$ then leads to a new $N_{2}$ decay channel, $N_{2} \rightarrow N_{1} S$, which causes the $N_{2}$ number density to depart more strongly from its would-be value in thermal equilibrium. At the same time, the new $N_{2}$ decay 

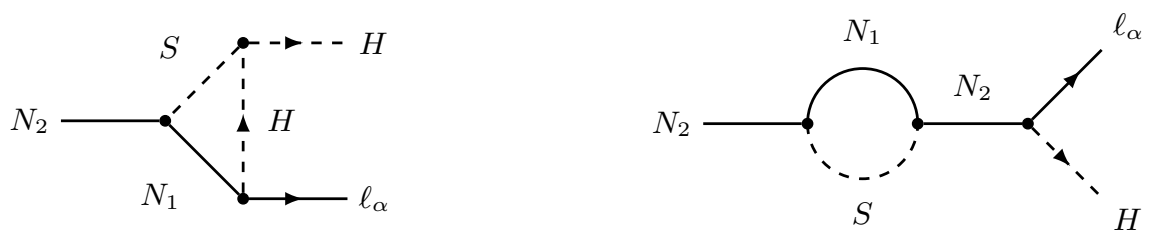

Figure 1. Scalar-singlet-mediated one-loop diagrams that enhance the amount of $C P$ violation in the decay $N_{2} \rightarrow \ell_{\alpha} H$.

channel also increases the amount of $C P$ violation in $N_{2}$ decays at loop level. Together, these two effects enhance the efficiency of leptogenesis, such that the BAU can be successfully generated in $N_{2}$ decays for RHN masses even below the $\mathrm{TeV}$ scale. The general principle behind this mechanism has been described for the first time by Le Dall and Ritz [46]. ${ }^{2}$ In this paper, we will build upon this earlier work and extend it in several directions. An important novel aspect of our analysis is that we perform a systematic scan of parameter space, which allows us to determine the dependence of the final asymmetry on the choice of parameter values in the neutrino sector. In particular, we derive a semianalytical fit function that allows us to reproduce the exact numerical result with high precision [see Eqs. (21) and (22)]. Moreover, we make sure that we only consider parameter values in the neutrino sector that are consistent with the most recent low-energy data on neutrino oscillations. This is accomplished by employing the Casas-Ibarra (CI) parametrization for the RHN Yukawa couplings to SM lepton-Higgs pairs [73].

The rest of this paper is organized as follows. In Sec. II, we will outline the characteristics of our model and discuss how the scalar $S$ affects some of the key quantities in the description of leptogenesis. In Sec. III, we will then turn to the modified Boltzmann equations and present our semianalytical fit function for the BAU. In Sec. IV, we will discuss further theoretical and phenomenological constraints on our scenario and highlight the viable regions in parameter space. Sec. V contains our conclusions, and in Appendix A, we collect some lengthy formulae.

\section{REAL-SCALAR-SINGLET EXTENSION OF THE TYPE-I SEESAW MECHANISM}

\section{A. Couplings and masses in the Lagrangian}

The starting point of our analysis is the type-I seesaw model featuring two heavy neutrinos $N_{1,2}$ and supple-

\footnotetext{
2 The first discussion of $N_{2}$-dominated leptogenesis in the standard type-I seesaw model can be found in Ref. [72].
}

mented by the interactions of the real scalar singlet, $\widetilde{S}$,

$$
\begin{aligned}
& -\mathcal{L} \supset\left[\widetilde{h}_{\alpha i} \ell_{\alpha} \widetilde{N}_{i} H+\frac{1}{2}\left(\widetilde{M}_{i j}+\widetilde{\alpha}_{i j} \widetilde{S}\right) \widetilde{N}_{i} \widetilde{N}_{j}+\text { H.c. }\right] \\
& +V(H, \widetilde{S}) ; \quad i, j=1,2 ; \quad \alpha=e, \mu, \tau .
\end{aligned}
$$

Here, we use a tilde above every quantity whose definition depends on the configuration of vacuum expectation values (VEVs) in the scalar sector. In Eq. (1), all the quantities equipped with a tilde are defined at high energies, where the scalar VEVs in our model are assumed to vanish. These fields and parameters should thus be considered as the fundamental input quantities of our model. The fields $H=\left(H^{+}, H^{0}\right)^{T}$ and $\ell_{\alpha}=\left(\nu_{\alpha}, \alpha_{L}\right)^{T}$ correspond to the SM Higgs doublet and the three SM lefthanded charged-lepton doublets, respectively. We denote the usual RHN-lepton-Higgs Yukawa matrix by $\widetilde{h}$, and $\widetilde{M}$ is a matrix of RHN input masses whose dynamical origin is left unspecified for the purposes of this work. The matrix $\widetilde{\alpha}$ characterizes the strength of the novel singlet-RHN Yukawa interactions. The scalar $\widetilde{S}$ will only be able to modify the dynamics of leptogenesis if the two matrices $\widetilde{M}$ and $\widetilde{\alpha}$ are not proportional to each other. That is, only if $\widetilde{M}$ and $\widetilde{\alpha}$ cannot be diagonalized simultaneously, will the scalar $\widetilde{S}$ induce flavor-changing neutral-current interactions among the heavy neutrinos that affect the efficiency of leptogenesis. We stress once more that both the RHN mass terms as well as the singlet-neutrino Yukawa interactions explicitly violate global lepton number.

The scalar potential, $V$, in Eq. (1) encompasses the SM Higgs potential as well as a number of terms involving the new scalar field, $\widetilde{S}$. We emphasize that the requirement of successful leptogenesis only leads to weak constraints on the overall shape of the scalar potential. The most important requirement is that the singlet scalar must share a trilinear coupling with two SM Higgs fields. Together with the new Yukawa interactions parametrized by $\widetilde{\alpha}$, this operator leads to a new source of $C P$ violation in $N_{2}$ decays at the one-loop level (see Fig. 1). One possibility is that the trilinear coupling is simply generated in the UV completion together with all other couplings of the scalar singlet $S$. Alternatively, one may assume that the singlet is odd under a $\mathbb{Z}_{2}$ symmetry of the scalar potential, and the trilinear coupling is generated via the Higgs-portal operator, $\widetilde{S}^{2}|H|^{2}$, as a consequence of spontaneous sym- 
metry breaking. In the rest of this paper, we will pursue this second, $\mathbb{Z}_{2}$-symmetric option for illustrative purposes. At the same time, we emphasize that the results for leptogenesis do not depend on this choice, but are valid for more generic scalar potentials containing a trilinear $\widetilde{S}|H|^{2}$ coupling. The restriction to the $\mathbb{Z}_{2}$-symmetric case, however, has several advantages. First of all, it comes with a smaller number of free input parameters in the renormalizable part of the Lagrangian. This will facilitate our discussion of the experimental and theoretical bounds on our model in Sec. IV. But in addition, a discrete $\mathbb{Z}_{2}$ symmetry may also provide us with some intuition regarding the smallness of the Yukawa couplings, $\widetilde{\alpha}$, which break this global $\mathbb{Z}_{2}$ symmetry explicitly. The $\mathbb{Z}_{2}$ symmetry in the scalar potential can therefore be at most an approximate symmetry that is explicitly broken by the singlet-neutrino Yukawa interactions. Conversely, this means that small values of the $\widetilde{\alpha}$ Yukawa couplings are symmetry-protected and hence natural in the sense of 't Hooft [74].

Let us now suppose that the singlet field in Eq. (1) does obtain a nonzero $\mathrm{VEV}, v_{S}$, at some high temperature above the EW phase transition, $T_{S} \gg T_{\text {ew }}$, and that leptogenesis occurs at energies in between these temperatures. It is then convenient to shift the scalar singlet by its VEV, $\widetilde{S} \rightarrow v_{S}+S$, and to perform a transformation on the heavy neutrinos in order to diagonalize their mass matrix $\widetilde{M}$. After these two steps, the Lagrangian reads

$$
\begin{aligned}
-\mathcal{L} & \supset\left[h_{\alpha i} \ell_{\alpha} N_{i} H+\frac{1}{2}\left(\delta_{i j} M_{j}+\alpha_{i j} S\right) N_{i} N_{j}+\text { H.c. }\right] \\
& +V(H, S)
\end{aligned}
$$

where we dropped all tildes to indicate that this Lagrangian is now valid at energies below the $\mathbb{Z}_{2}$-symmetry breaking scale. This Lagrangian encodes the RHN interactions that are relevant for leptogenesis. It is interesting to note that the masses and couplings in Eq. (2) still do not fully coincide with the respective parameters at low energies. The reason for this is that the singlet $\mathrm{VEV}, v_{S}$, is expected to receive corrections during the EW phase transition, $v_{S} \rightarrow v_{S}^{0}$, which necessitates yet another shift in the scalar singlet, $S \rightarrow v_{S}^{0}-v_{S}+s$, as well as yet another diagonalization of the RHN mass matrix. ${ }^{3}$

After EW symmetry breaking, the final low-energy Lagrangian in unitary gauge obtains the following form,

$$
\begin{aligned}
-\mathcal{L} & \supset\left[\frac{h_{\alpha i}^{0}}{\sqrt{2}} \nu_{\alpha} N_{i}^{0} h+\frac{1}{2}\left(\delta_{i j} M_{j}^{0}+\alpha_{i j}^{0} s\right) N_{i}^{0} N_{j}^{0}+\text { H.c. }\right] \\
& +V(h, s)
\end{aligned}
$$

\footnotetext{
3 These corrections are related to $\alpha_{i j}$ and therefore small in our scenario, so we will neglect them in the analysis of leptogenesis.
}

where $h$ denotes the physical SM Higgs boson with a mass of $125 \mathrm{GeV}$. The Lagrangian in Eq. (3) sets the stage for the type-I seesaw mechanism. Upon integrating out the heavy sterile neutrinos, the light SM neutrinos acquire a Majorana mass matrix, $m_{\nu}$, of the following form [4-8],

$$
m_{\nu}=-m_{D} D_{N}^{-1} m_{D}^{T},
$$

where $m_{D}=h^{0} / \sqrt{2} v_{\text {ew }}$ denotes the neutrino Dirac mass matrix and $D_{N}=\operatorname{diag}\left(M_{1}^{0}, M_{2}^{0}\right)$ is the diagonal heavyneutrino Majorana mass matrix. The light-neutrino Majorana mass matrix, $m_{\nu}$, can be diagonalized by acting on it with a unitary transformation, $D_{\nu}=U^{T} m_{\nu} U$. In the flavor basis where the charged-lepton Dirac mass matrix is diagonal, the matrix $U$ coincides with the Pontecorvo-MakiNakagawa-Sakata (PMNS) lepton mixing matrix [75, 76]. According to the CI parametrization [73], the Yukawa matrix, $h^{0}$, can then be partially reconstructed based on the information contained in $U, D_{\nu}$, and $D_{N}$ :

$$
\frac{h^{0}}{\sqrt{2}}=\frac{i}{v_{\mathrm{ew}}} U^{*} D_{\nu}^{1 / 2} R D_{N}^{1 / 2}
$$

For only two heavy sterile neutrinos, $R$ is a complex $3 \times 2$ rotation matrix that satisfies $R^{T} R=\mathbb{1}$ and that can be parametrized in terms of one complex rotation angle $z$. Besides that, the right-hand side of Eq. (5) also depends on the mixing angles $\theta_{12}, \theta_{13}$, and $\theta_{23}$, the phases $\delta_{\mathrm{CP}}$ and $\alpha_{\mathrm{CP}}$ in the PMNS matrix $U$, the mass splittings $\Delta m_{\mathrm{sol}}^{2}$ and $\Delta m_{\text {atm }}^{2}$ in the light-neutrino mass spectrum, and the mass eigenvalues $M_{1}^{0}$ and $M_{2}^{0}$ of the two heavy sterile neutrinos. In this paper, we shall neglect all flavor effects in the computation of the final BAU for simplicity. As we will see, the dynamics of leptogenesis will thus be controlled by the entries of the Hermitian matrix $h^{0 \dagger} h^{0}$,

$$
\frac{h^{0 \dagger} h^{0}}{2}=\frac{1}{v_{\mathrm{ew}}^{2}} D_{N}^{1 / 2} R^{\dagger} D_{\nu} R D_{N}^{1 / 2},
$$

which is independent of the PMNS matrix. In our numerical analysis in Sec. III, we will use the best-fit values for $\Delta m_{\text {sol }}^{2}$, and $\Delta m_{\text {atm }}^{2}$ according to the most recent global-fit analysis presented by the NuFIT collaboration [77]. The RHN mass eigenvalues $M_{1}^{0}$ and $M_{2}^{0}$ as well as the complex rotation angle $z$ will be treated as free input parameters.

\section{B. $C P$ asymmetry in RHN decays}

The decay of the heavy Majorana neutrinos into SM lepton-Higgs pairs, $N_{i} \rightarrow \ell_{\alpha} H, \ell_{\alpha}^{\dagger} H^{\dagger}$, violates $C P$ invariance. The amount of $C P$ violation in these decays is conveniently measured by the $C P$-asymmetry parameters

$$
\varepsilon_{i}=\frac{\sum_{\alpha}\left[\Gamma\left(N_{i} \rightarrow \ell_{\alpha} H\right)-\Gamma\left(N_{i} \rightarrow \ell_{\alpha}^{\dagger} H^{\dagger}\right)\right]}{\sum_{\alpha}\left[\Gamma\left(N_{i} \rightarrow \ell_{\alpha} H\right)+\Gamma\left(N_{i} \rightarrow \ell_{\alpha}^{\dagger} H^{\dagger}\right)\right]} .
$$


In the standard type-I seesaw model without any extra scalar DOFs, evaluating this expression results in [78]

$$
\varepsilon_{i}^{0}=\frac{1}{8 \pi\left(h^{\dagger} h\right)_{i i}} \sum_{j \neq i} \operatorname{Im}\left[\left(h^{\dagger} h\right)_{j i}^{2}\right] \mathcal{F}\left(\frac{M_{j}}{M_{i}}\right),
$$

with the loop function $\mathcal{F}$ being defined as follows:

$$
\mathcal{F}(x)=x\left[1+\left(1+x^{2}\right) \ln \left(\frac{x^{2}}{x^{2}+1}\right)-\frac{1}{x^{2}-1}\right] .
$$

This result needs to be compared to the $C P$-asymmetry parameters $\varepsilon_{1,2}$ in the presence of the scalar singlet, $S$. The decay $N_{1} \rightarrow N_{2} S$ is kinematically forbidden, which is why the parameter $\varepsilon_{1}^{0}$ remains unchanged. The parameter $\varepsilon_{2}^{0}$, however, receives further contributions from the oneloop vertex (v) and self-energy (s) diagrams in Fig. 1,

$$
\varepsilon_{1}=\varepsilon_{1}^{0}, \quad \varepsilon_{2}=\varepsilon_{2}^{0}+\varepsilon_{2}^{\mathrm{v}}+\varepsilon_{2}^{\mathrm{s}} .
$$

An explicit computation of the extra terms yields [46]

$$
\begin{aligned}
8 \pi\left(h^{\dagger} h\right)_{22} \varepsilon_{2}^{\mathrm{v}} & =\operatorname{Im}\left[\left(h^{\dagger} h\right)_{12} \beta_{2} \alpha_{21}\right] \mathcal{F}_{21, R}^{\mathrm{v}} \\
& +\operatorname{Im}\left[\left(h^{\dagger} h\right)_{12} \beta_{2} \alpha_{21}^{*}\right] \mathcal{F}_{21, L}^{\mathrm{v}}, \\
8 \pi\left(h^{\dagger} h\right)_{22} \varepsilon_{2}^{\mathrm{s}} & =\operatorname{Im}\left[\left(h^{\dagger} h\right)_{12} \alpha_{21} \alpha_{11}\right] \mathcal{F}_{211, R R}^{\mathrm{s}} \\
& +\operatorname{Im}\left[\left(h^{\dagger} h\right)_{12} \alpha_{21}^{*} \alpha_{11}\right] \mathcal{F}_{211, R L}^{\mathrm{s}} \\
& +\operatorname{Im}\left[\left(h^{\dagger} h\right)_{12} \alpha_{21} \alpha_{11}^{*}\right] \mathcal{F}_{211, L R}^{\mathrm{s}} \\
& +\operatorname{Im}\left[\left(h^{\dagger} h\right)_{12} \alpha_{21}^{*} \alpha_{11}^{*}\right] \mathcal{F}_{211, L L}^{\mathrm{s}},
\end{aligned}
$$

where $\beta_{2}=\mu / M_{2}$ is related to the dimensionful coupling $\mu$ of the trilinear $S|H|^{2}$ operator in the scalar potential,

$$
V(H, S) \supset \mu S|H|^{2} .
$$

General expressions for the loop functions in Eq. (11) can be found in Appendix A. The indices $R$ and $L$ indicate whether a given contribution to $\varepsilon_{2}$ stems from the interaction of the scalar field, $S$, with a pair of right-chiral or left-chiral neutrino spinor fields, respectively.

The expressions in Eq. (11) illustrate the importance of the off-diagonal singlet-neutrino Yukawa coupling, $\alpha_{21}$. Only if this coupling is nonzero will the scalar field $S$ mediate flavor-changing neutral-current interactions among the heavy neutrinos and hence lead to an enhanced $C P$ asymmetry in $N_{2}$ decays. As we will see in Sec. III, an important feature of our scenario is that this gain in $C P$ asymmetry is not counteracted by a correspondingly stronger washout. The reason for this is that the decay $N_{2} \rightarrow N_{1} S$ does not involve the usual SM lepton-Higgs pairs and thus does not increase the strength of the usual asymmetry washout by inverse decays. This needs to be contrasted with the situation in standard thermal leptogenesis, where both the amount of $C P$ asymmetry and

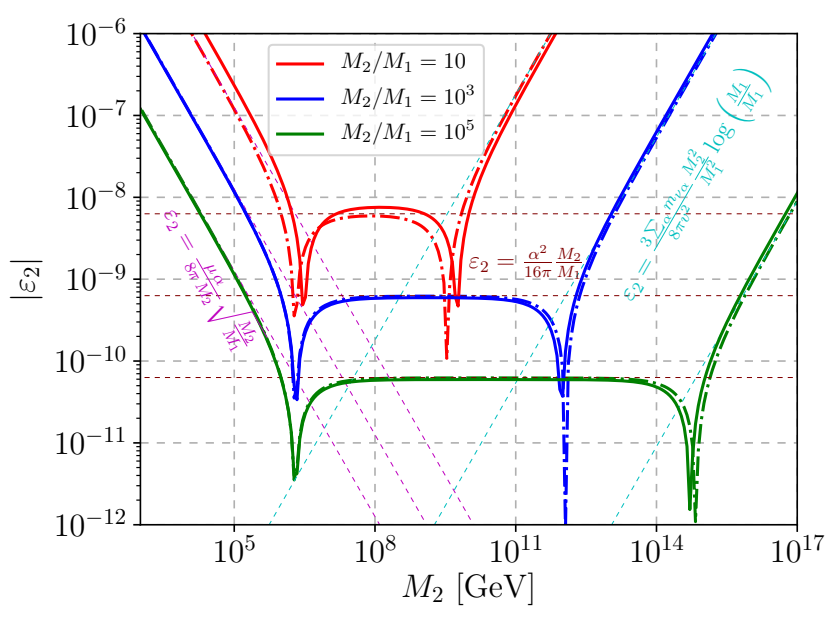

Figure 2. $C P$-asymmetry parameter $\varepsilon_{2}$ in Eq. (8) as a function of the heavy RHN mass. Here, $\alpha_{i j}=10^{-3}, \mu=1 \mathrm{TeV}$, and $m_{S} \ll M_{2}$. The solid lines show the exact result; the dashed lines indicate the estimates in Eq. (13) (see also Ref. [46]); and the dot-dashed lines represent the sums of the respective estimates. The exact asymmetry changes sign depending on the signs of the couplings and CI angle.

the washout efficiency are controlled by the same combination of couplings, i.e. by the same products of Yukawa couplings of the form $h_{\alpha i}^{*} h_{\beta j}$.

For a sufficiently hierarchical RHN mass spectrum, the $C P$-asymmetry parameters $\varepsilon_{2}^{\mathrm{v}}$ and $\varepsilon_{2}^{\mathrm{s}}$ in Eq. (11) can be considerably simplified. For $M_{2} \gg M_{1}$, one obtains

$$
\begin{aligned}
& \varepsilon_{2}^{\mathrm{v}} \approx-\frac{\left|\beta_{2} \alpha_{21}\right|}{8 \pi} \sqrt{\frac{M_{1}}{M_{2}}}\left(1-\frac{m_{S}^{2}}{M_{2}^{2}}\right), \\
& \varepsilon_{2}^{\mathrm{s}} \approx \frac{\left|\alpha_{21} \alpha_{11}\right|}{16 \pi} \sqrt{\frac{M_{1}}{M_{2}}}\left(1-\frac{m_{S}^{2}}{M_{2}^{2}}\right)^{2} .
\end{aligned}
$$

In Fig. 2, we compare these estimates (dashed lines) with the corresponding exact expressions in Eqs. (11) (solid lines). As evident from this figure, the new contributions to the $C P$ asymmetry are dominant for comparatively low $N_{2}$ masses. The new vertex contribution $\varepsilon_{2}^{\mathrm{v}}$, e.g., leads to a strong enhancement of the total $C P$ asymmetry for $N_{2}$ masses less than $\mathcal{O}\left(10^{6}\right) \mathrm{GeV}$. Unfortunately, this increase in the $C P$ asymmetry will not enable us to lower the energy scale of leptogenesis to arbitrarily small RHN masses. As we will discuss in Sec. III B, 2-to-2 scattering processes will eventually give rise to new sources of washout and thus limit the efficiency of leptogenesis. From Eq. (13), one can also see that at some point a larger RHN mass scale will lower $\varepsilon_{2}^{\mathrm{v}}$ below $\varepsilon_{2}^{\mathrm{s}}$, and the contribution becomes independent of $\mu$. Finally, the $C P$ asymmetry will revert to grow proportional to the RHN masses, and we enter the standard type-I regime. 


\section{BOLTZMANN EQUATIONS AND A SEMIANALYTICAL SOLUTION}

To gain a physical understanding of the scalar-assisted leptogenesis induced by $N_{2}$ decays, we consider a coupled set of classical Boltzmann equations for the normalized number densities, $N_{N_{i}}$. These capture the decays and inverse decays of RHNs into leptons and the Higgs doublet, as well as the corresponding processes for the decay $N_{2} \rightarrow N_{1} S$. Furthermore, we include the dominant washout effect induced by the $\Delta L=2$ scatterings $N_{i} N_{j} \rightarrow H H$ mediated by the singlet. The relevant equations read [46]

$$
\begin{aligned}
\frac{\mathrm{d} N_{N_{2}}}{\mathrm{~d} z}= & -\left(D_{2}+D_{21}\right) \Delta_{N_{2}}+D_{21} \Delta_{N_{1}} \\
& -\Delta_{N_{1} N_{2}} S_{N_{1} N_{2} \rightarrow H H} \\
& -\Delta_{N_{2} N_{2}} S_{N_{2} N_{2} \rightarrow H H} \\
\frac{\mathrm{d} N_{N_{1}}}{\mathrm{~d} z}= & -\left(D_{1}+D_{21}\right) \Delta_{N_{1}}+D_{21} \Delta_{N_{2}} \\
& -\Delta_{N_{1} N_{2}} S_{N_{1} N_{2} \rightarrow H H} \\
& -\Delta_{N_{1} N_{1}} S_{N_{1} N_{1} \rightarrow H H}, \\
\frac{\mathrm{d} N_{B-L}}{\mathrm{~d} z}= & -\sum_{i=1}^{2} \varepsilon_{i} D_{i} \Delta_{i}-W N_{B-L},
\end{aligned}
$$

with the temperature-dependent quantities

$$
\begin{gathered}
\Delta_{N_{i}}(z) \equiv \frac{N_{N_{i}}(z)}{N_{N_{i}}^{\mathrm{eq}}(z)}-1, \\
\Delta_{N_{i} N_{j}} \equiv \frac{N_{N_{i}} N_{N_{j}}}{N_{N_{i}}^{\mathrm{eq}} N_{N_{j}}^{\mathrm{eq}}}-1, \\
N_{N_{i}}^{\mathrm{eq}}(z)=\frac{z_{i}^{2}}{2} \mathcal{K}_{2}\left(z_{i}\right) \\
D_{i}(z)=K_{i} z \frac{\mathcal{K}_{1}\left(z_{i}\right)}{\mathcal{K}_{2}\left(z_{i}\right)} N_{N_{i}}^{\mathrm{eq}}(z), \\
D_{21}(z)=K_{21} z \frac{\mathcal{K}_{1}\left(z_{2}\right)}{\mathcal{K}_{2}\left(z_{2}\right)} N_{N_{2}}^{\mathrm{eq}}(z), \\
W(z)=\sum_{i} \frac{1}{4} K_{i} z_{i}^{3} \mathcal{K}_{1}\left(z_{i}\right) .
\end{gathered}
$$

Here, $\mathcal{K}_{a}$ denotes the $a$-th modified Bessel function of the second kind, and $z_{i} \equiv M_{i} / T$ is the inverse dimensionless temperature for the $i$-th RHN, with $z \equiv z_{1}$. The relevant decay parameters read, respectively,

$$
K_{i} \equiv \frac{\Gamma\left(N_{i} \rightarrow L H\right)}{H\left(T=M_{i}\right)}=\sqrt{\frac{45}{64 \pi^{5} g_{*}}} \frac{M_{\mathrm{Pl}}}{v_{\mathrm{ew}}^{2}}\left(\mathcal{M}_{\nu}^{R}\right)_{i i},
$$

with the Hubble rate $H=\sqrt{8 \pi^{3} g_{*} / 90} T^{2} / M_{\mathrm{Pl}}$. The quantity $\mathcal{M}_{\nu}^{R}$ emerges from the CI parametrization for the neutrino Yukawa matrix, Eq. (6), and is given by

$$
\mathcal{M}_{\nu}^{R} \equiv R^{\dagger} D_{\nu} R
$$

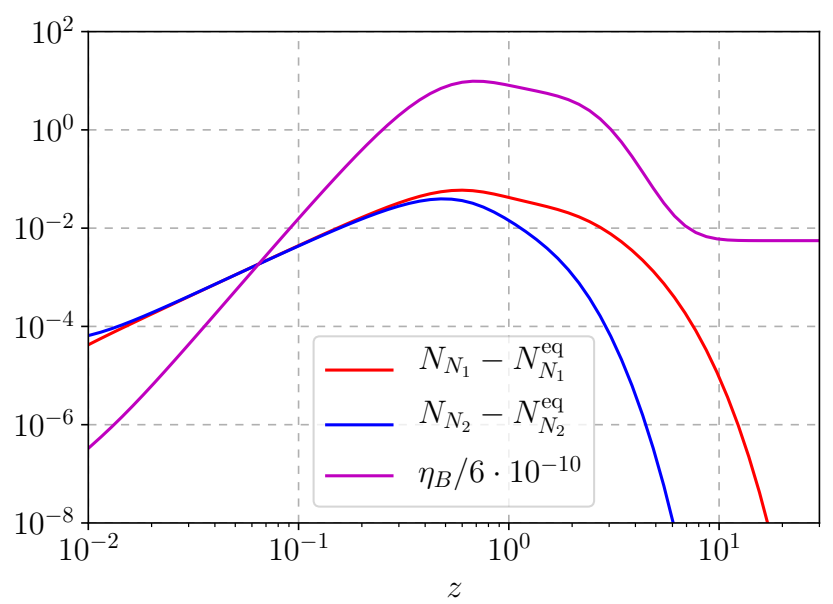

Figure 3. Sample evolution of RHN number density and baryon asymmetry as a function of $z \equiv z_{1}=M_{1} / T$. For this scenario, we fixed the parameters as follows: $M_{1}=2 \mathrm{TeV}$, $M_{2}=6 \mathrm{TeV}, m_{S}=\mu=500 \mathrm{GeV}, m_{H}=0$, and $\alpha_{i j}=10^{-3}$. The CI rotation angle $z$ is fixed such that $K_{1}=12, K_{2}=51$.

where $R$ is the aforementioned arbitrary complex matrix that satisfies $R^{T} R=\mathbb{1}$. Note that the decay parameters $K_{i}$ are independent of the RHN masses and that, for two RHNs, their values are bounded to be larger than $K_{i} \gtrsim 8$, i.e., we are always in the regime of strong washout. The decay parameter of the new decay channel reads

$$
K_{21} \equiv \frac{\Gamma\left(N_{2} \rightarrow N_{1} S\right)}{H\left(T=M_{2}\right)},
$$

where the decay width for $N_{2} \rightarrow N_{1} S$ is given by [46]

$$
\Gamma\left(N_{2} \rightarrow N_{1} S\right)=\frac{\left|\alpha_{12}\right|^{2} M_{2}}{16 \pi}\left[\left(1+r_{12}\right)^{2}-\sigma_{2}\right] \sqrt{\delta_{12}},
$$

with

$$
\begin{aligned}
r_{i j} & \equiv\left(M_{i} / M_{j}\right)^{2}, \quad \sigma_{i} \equiv m_{S}^{2} / M_{i}^{2}, \\
\delta_{i j} & \equiv\left(1-r_{i j}-\sigma_{j}\right)^{2}-4 r_{i j} \sigma_{j} .
\end{aligned}
$$

Typically, the new decay parameter is very large, e.g., $K_{21} \sim 10^{6}$ for $M_{2}=1 \mathrm{TeV}$ and $\alpha_{12}=10^{-3}$. This is crucial for the RHN number densities to display a significant deviation from their equilibrium form. We will discuss the impact of the scattering terms, $S_{N_{i} N_{j} \rightarrow H H}$, and give their explicit expressions in Sec. III B. For the rest of this section, we will assume that all scattering terms in Eqs. (14) are negligible.

Solving this set of coupled differential equations, we see that the genesis of a lepton number asymmetry proceeds in two steps; cf. Fig. 3: First, the $N_{2}$ decays into leptons and anti-leptons build up a large lepton or $B-L$ asymmetry around temperatures $z_{2} \lesssim 1$. This is driven mostly 
by the enhanced $C P$ asymmetry due to the new decay channels. Subsequently, once $N_{1}$ drops out of thermal equilibrium, around $z_{1} \sim 1$, the analogous decay for $N_{1}$ occurs; however, due to the small $C P$ asymmetry, this effect is negligible for $N_{1}$ masses $\lesssim 10^{6} \mathrm{GeV}$ [46]. At the same time, inverse $N_{1}$ decays wash out the asymmetry previously built up by the decay of $N_{2}$, reducing the final $B-L$ asymmetry exponentially, as one can infer from the last term in Eq. (14c) and the rapid decrease of the purple curve in Fig. 3 before becoming constant. The overall magnitude of the washout is, however, smaller than in the standard type-I seesaw framework, as a deviation of the $N_{1}$ number density from thermal equilibrium immediately leads to a comparable deviation of the $N_{2}$ number density from its equilibrium value by virtue of the large $K_{21}$. Thus, strong washout is always accompanied by more $C P$ violating decays, enhancing the lepton asymmetry and counteracting the washout.

The final $B-L$ asymmetry is subsequently converted into the baryon asymmetry via $\mathrm{EW}$ sphalerons, which become inefficient below a temperature $T_{\mathrm{sph}}=131 \mathrm{GeV}$ [79], and therefore the relevant asymmetry needs to be generated at temperatures above $T_{\mathrm{sph}}$. Note, however, that an asymmetry generated just before sphaleron interactions freeze-out will persist without being subject to washout (which affects only the lepton sector). This has already been used in Ref. [46] to find low-mass scenarios with $M_{1}<T_{\mathrm{sph}} \lesssim M_{2}$. The final baryon-to-photon ratio is given by $\eta_{B} \simeq 0.01 N_{B-L}(z=\infty)$, which accounts for the sphaleron conversion and entropy production after the generation of the lepton asymmetry and is already included in Fig. 3. However, we remark that, while the example on display highlights the distinct regimes, the resulting asymmetry is two orders of magnitude lower than the measured value. This indicates that some additional source of $C P$ violation is needed - limited by scattering processes; cf. Sec. IIIB. Alternatively, the masses must be of similar magnitude in order to suppress the washout.

By numerical integration of Eqs. (14), one readily obtains a value for $N_{B-L}(z=\infty)$; however, the physical insight is limited due to the extended parameter space of the model. The subsequent subsections are dedicated to the development of an analytical understanding of these mechanisms and comparing them to the exact, numerical results including also the scattering terms. Eventually, we will use these results to identify parameter regions that successfully explain the observed baryon asymmetry.

\section{A. A semianalytical fit}

Let us try to simplify the treatment of leptogenesis by finding an approximation to the exact solution of the
Boltzmann equations in Eq. (14). As we observed in the scenario shown in Fig. 3, there was not enough $C P$ violation and/or too much washout. While the $C P$ asymmetry can always be increased by raising $\mu$, cf. Eqs. (11) and (13), eventually $\Delta L=2$ scattering processes will become important and reduce the final asymmetry by increasing the washout. Thus, we seek to reduce the washout by reducing the mass splitting of the RHNs. As long as the $\Delta L=2$ scattering processes are irrelevant, the final baryon asymmetry will be given by [22]

$$
\eta_{B} \simeq 0.01 \kappa_{2}\left(K_{2}, r\right) \varepsilon_{2} \times e^{-\omega\left(K_{1}, r\right)},
$$

where $r \equiv r_{12}=\left(M_{1} / M_{2}\right)^{2}$. This holds true, even in the non-hierarchical regime, because only the $N_{2}$ decays display significant $C P$ violation via Eqs. (11), and at the same time only $N_{1}$ decays wash out the asymmetry. While simple approximations assuming a large hierarchy between the RHN masses yield $\omega=3 \pi / 8 K_{1}$ and $\kappa_{2}=$ $2 / z_{B}\left(K_{2}\right)$ with $z_{B}\left(K_{2}\right)=2+4 K_{2}{ }^{0.13} e^{-2.5 / K_{2}}$ [22], these will become more complicated as the masses are of the same order - however, non-degenerate (see also Ref. [80], which studied a similar setting for the type-I seesaw case). Allowing these functions to take a more general form, we find for the efficiency factor, $\kappa_{2}$, and the washout function, $\omega$, a good fit to be given by:

$$
\begin{aligned}
& \kappa_{2}\left(K_{2}, r\right)= \frac{2}{z_{B}\left(K_{2}\right)} \frac{K_{2}^{0.44} r^{-0.2}}{0.3 K_{2}+1.6 r^{2.6}} \\
& \omega\left(K_{1}, r\right)=\frac{3 \pi}{8}\left[-K_{1}^{0.5}\left(1.4 r^{2}-2.1 r+0.8\right) \ln (r)\right. \\
&\left.\quad+1.2 K_{1}^{0.2}\right]
\end{aligned}
$$

which are functions of the decay constants, $K_{1,2}$, and the mass ratio $r$. In Fig. 4, we show in solid contour lines the numerically calculated efficiency factor and washout exponent, which are determined in terms of the maximal $B-L$ asymmetry, $N_{B-L}^{\max }=\max _{z} N_{B-L}(z)$, as $\kappa_{2}=N_{B-L}^{\max } / \varepsilon_{2} \geq 0$ and $\omega=\ln N_{B-L}^{\max } / N_{B-L}(\infty) \geq 0$. These are compared to the above fit functions shown in dashed lines. The colors indicate the relative deviation of the numerically determined efficiency and washout compared to the fits, and we find that they provide a good approximation for the values of $K_{1,2}$ and $r$ of interest to us with relative deviations not larger than about $10 \%$.

\section{B. Washout effects due to scattering processes}

The complete set of Boltzmann equations including different scattering terms was given in Ref. [46]. The dominant washout effect is induced by the scatterings $N_{i} N_{j} \rightarrow H H$ mediated via the scalar singlet. While the asymmetry factors can be enhanced by increasing 

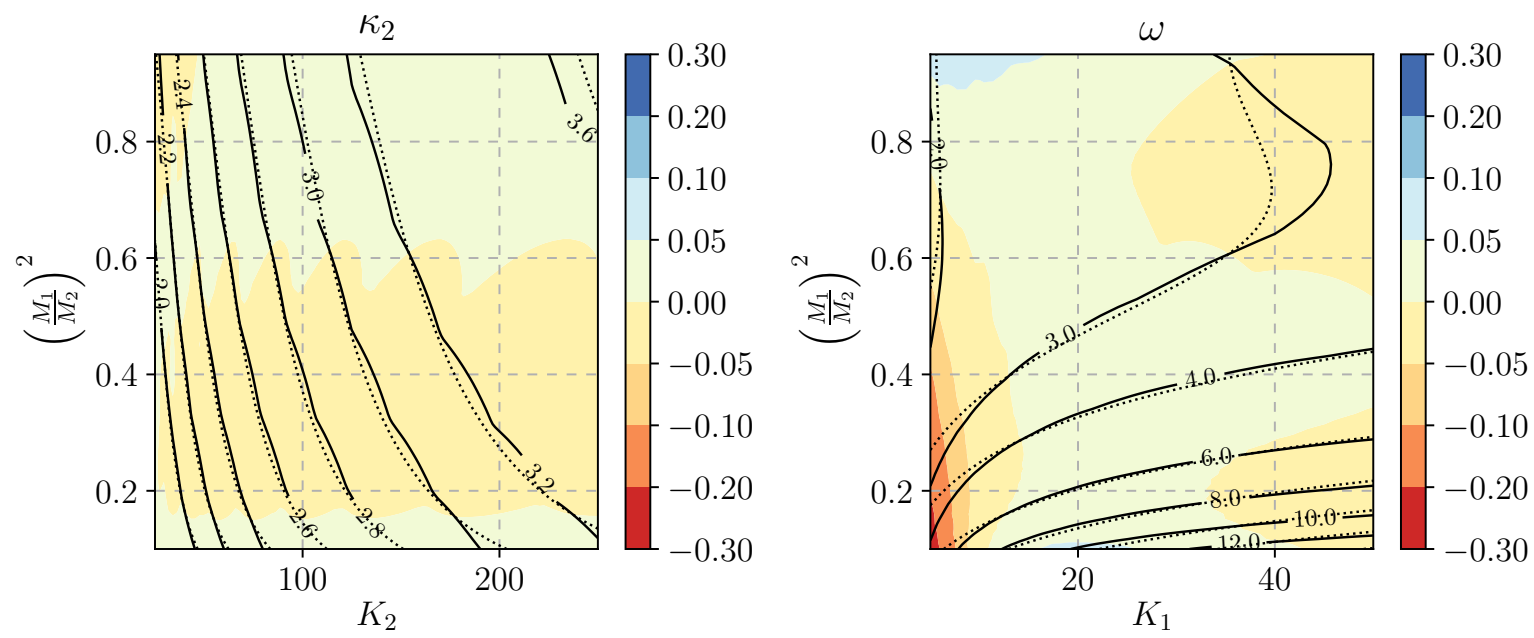

Figure 4. Comparison of the efficiency factor $\kappa_{2}$ and the washout exponent $\omega$ for the numerical scan (solid lines) and the fit functions presented in Eqs. $(21,22)$ (dashed lines). The relative deviation is shown in color coding and is less than $10 \%$ for most of the parameter values of interest.

the trilinear coupling, $\mu$, this simultaneously enhances the scatterings, as can be seen from the scattering cross section [62]

$$
\sigma_{N_{i} N_{j} \rightarrow H H}=\frac{\left|\left(\alpha \alpha^{\dagger}\right)_{j i}\right|^{2} \mu^{2}}{8 \pi} \frac{s-\left(M_{i}+M_{j}\right)^{2}}{\left(s-m_{S}^{2}\right)^{2} \sqrt{\delta_{M_{i}, M_{j}}}},
$$

where

$$
\delta_{M_{i}, M_{j}}=\left(s-M_{i}^{2}-M_{j}^{2}\right)^{2}-4 M_{i}^{2} M_{j}^{2} .
$$

The scattering functions, $S_{N_{i} N_{j} \rightarrow H H}$, in Eqs. (14) can be written in terms of the scattering cross sections as [46]

$$
\begin{aligned}
& S_{N_{i} N_{j} \rightarrow H H} \equiv \frac{M_{i}}{64 \pi^{2} H\left(T=M_{i}\right)} \\
& \times \int_{w_{\min }}^{\infty} \mathrm{d} w \sqrt{w} K_{1}(\sqrt{w}) \hat{\sigma}_{N_{i} N_{j} \rightarrow H H}\left(\frac{w M_{i}^{2}}{z_{i}^{2}}\right),
\end{aligned}
$$

where $w_{\min }=\left(M_{i}+M_{j}\right)^{2}$, and

$$
\hat{\sigma}_{N_{i} N_{j} \rightarrow H H}=\frac{1}{s} \delta_{M_{i}, M_{j}} \sigma_{N_{i} N_{j} \rightarrow H H} .
$$

Comparing the analytical estimates and the exact result including these scattering terms, we find that the results agree well as long as

$$
\mu<\mu_{*} \simeq M_{1} \times\left(\frac{0.5}{\delta_{M}}\right)\left(\frac{10^{-3}}{\alpha}\right),
$$

where

$$
\delta_{M} \equiv \frac{M_{2}-M_{1}}{M_{1}}
$$

and we have taken all $\alpha_{i j}$ equal and denote them here and in the following collectively by $\alpha$. Moreover, we observe that, since these scattering processes are relevant only to the washout, the regime $\mu>\mu_{*}$ will exhibit less $B-L$ asymmetry than the regime for which Eq. (27) holds. Thus, the baryon asymmetry is maximal for a value $\mu \sim \mu_{*}$, where the trilinear coupling is large enough to yield sufficient $C P$ asymmetry and yet not too large for scatterings to contribute significantly to the washout. This interplay is shown in Fig. 5, where we compare in red (left scale) the final baryon asymmetries obtained via the semianalytical fit Eqs. $(21,22)$ (dotted line) and the exact numerical solution, including washout due to scattering (solid line). The dashed blue curve (right scale) shows the ratio of the numerical result with and without scatterings. When this ratio becomes $\mathcal{O}(1)$, scatterings tend to increase the washout and consequently the two red curves deviate. The scale $\mu_{*}$ is therefore chosen such that for $\mu<\mu_{*}$ scatterings are negligible and good agreement between numerics and Eqs. $(21,22)$ is achieved.

\section{VIABLE PARAMETER SPACE FOR LOW-SCALE LEPTOGENESIS}

As a concrete and minimal example, we consider the $\mathbb{Z}_{2}$-symmetric scalar potential with a real singlet scalar:

$$
\begin{aligned}
V(H, \widetilde{S}) & =\widetilde{m}_{H}^{2}|H|^{2}+\frac{1}{2} \widetilde{m}_{S}^{2} \widetilde{S}^{2}+\lambda_{H}|H|^{4} \\
& +\frac{1}{2} \lambda_{S H} \widetilde{S}^{2}|H|^{2}+\frac{1}{4} \lambda_{S} \widetilde{S}^{4} .
\end{aligned}
$$



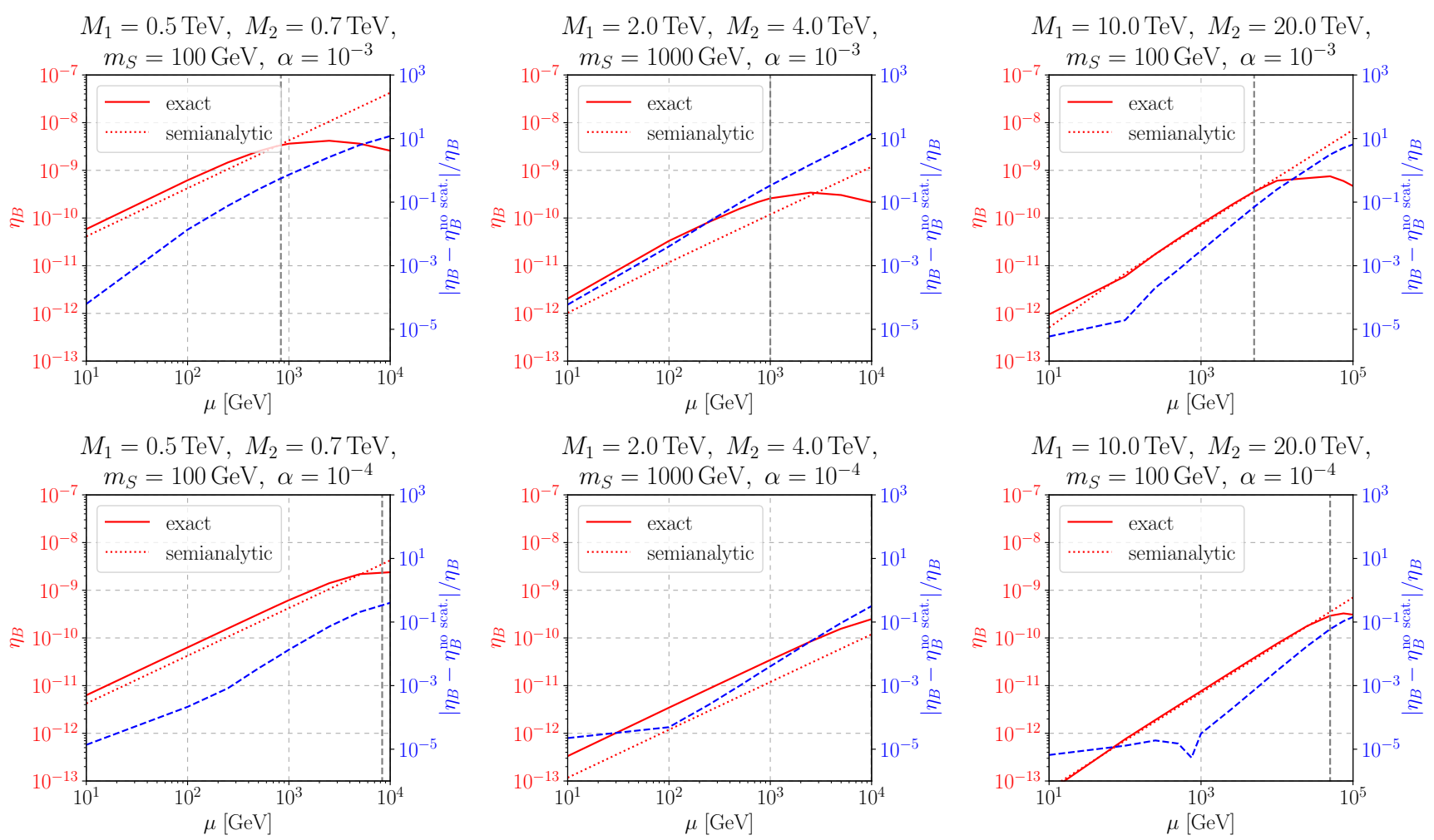

Figure 5. Comparison of the baryon asymmetry obtained via the solution of the full set of Boltzmann equations including scatterings (solid red line) and our estimate in Eq. (22) (dashed red line) for different masses (columns) and couplings $\alpha_{i j}=10^{-3}, 10^{-4}$ (rows) as indicated above each plot. We observe that, as long as $\mu<\mu_{*}$ [Eq. (27)], the results agree well, and the true baryon asymmetry peaks for a value of $\mu$ close to $\mu_{*}$ (dashed vertical line). The blue curve indicates the ratio of the final baryon asymmetry obtained numerically by solving the Boltzmann equations with and without the scatterings. The scatterings are important when this ratio grows to be $\mathcal{O}(1)$, which in turn defines $\mu_{*}$. We checked that the approximation is valid for a larger range of parameters, but do not show this here explicitly.

Here, we also adopted the notation of Sec. II that the vacuum-dependent quantities are equipped with a tilde in the high-temperature phase, where all VEVs vanish, and the quantities during leptogenesis are denoted without a tilde. We first employ several theoretical and experimental constraints on parameter space: For the above perturbative treatment to be valid, the quartic couplings need to be small, naively $\lambda_{H},\left|\lambda_{S H}\right|<4 \pi$. We, however, adopt a more conservative bound $\left|\lambda_{i}\right|<3$ in the light of perturbative unitarity bounds [81]. Furthermore, to ensure that the scalar potential is bounded from below, we have to require that $\lambda_{H}, \lambda_{S} \geq 0$ and $\lambda_{S H}>-2 \sqrt{\lambda_{H} \lambda_{S}}$, and the corresponding excluded region is indicated in Fig. 6 by $\lambda_{i}<\lambda_{i}^{\text {min,vac }}$.

From the Higgs signal-strength measurements, the mixing angle between the $125-\mathrm{GeV}$ Higgs and the singlet is bounded by $\sin \theta \lesssim 0.3[66,82]$. In addition, we include limits on the mixing angle from searches for resonant scalar-singlet production at the LHC. The limits we show correspond to those reported by the CMS collaboration based on $36 \mathrm{fb}^{-1}$ of data at $13 \mathrm{TeV}[71,83]$. These searches give a stronger limit than the Higgs signal-strength measurements for scalar-singlet masses $m_{S} \lesssim 800 \mathrm{GeV}$. Furthermore, for a very light scalar, $m_{S}<m_{h} / 2$, the Higgs can decay into two singlets, and the invisible Higgs decays are currently bounded by $\mathrm{BR}(h \rightarrow$ inv $) \leq 0.23$ [84, 85]. The width for the Higgs decay $h \rightarrow S S$ is given by

$$
\Gamma_{h \rightarrow S S}=\frac{\lambda_{S H}^{2} v_{\mathrm{ew}}^{2}}{32 \pi m_{h}} \sqrt{1-\frac{4 m_{S}^{2}}{m_{h}^{2}}},
$$

while the Higgs total decay width to the visible SM channels is $\Gamma_{h}=4.07 \mathrm{MeV}$ for $m_{h}=125 \mathrm{GeV}$ [86].

For the low-scale leptogenesis mechanism to work, the scalar singlet must acquire a nonzero $\operatorname{VEV}\left(v_{S}>0\right)$ to induce the trilinear coupling $\mu S|H|^{2}$, and the condition that the decay $N_{2} \rightarrow N_{1} S$ has to be kinematically allowed constrains the singlet mass during leptogenesis from above, $m_{S}<M_{1}-M_{2}$. In addition, we need the asymmetry to be generated before the $\mathrm{EW}$ phase transition, i.e. the phase transition in the singlet direction has to happen well 

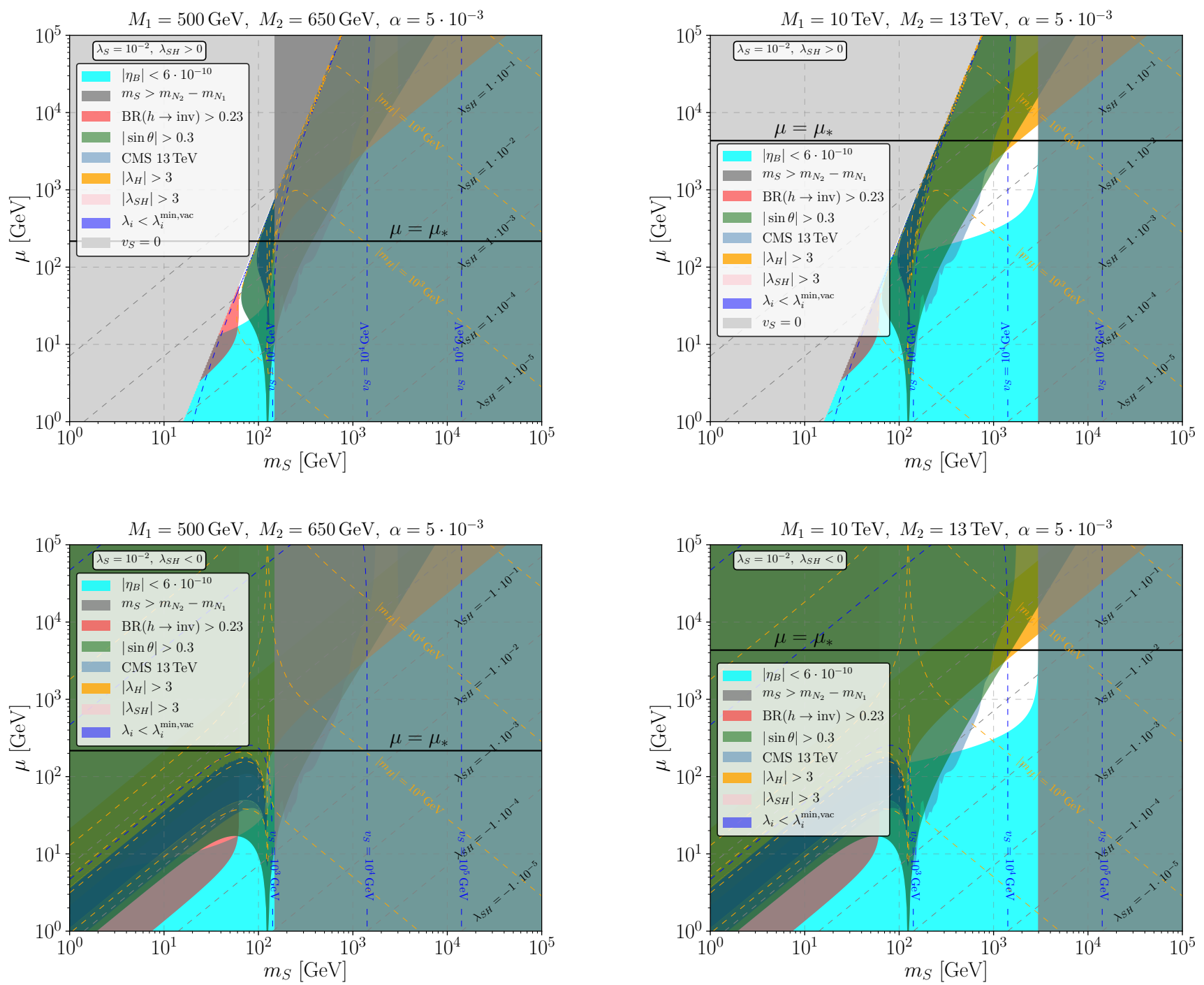

Figure 6. Parameter scan obtained using our semianalytical approximation. We fixed the mass ratio, such that $\delta=0.3$, which maximizes the resulting asymmetry. The CI angle is fixed such that $K_{1}=12, K_{2}=51$. Raising the mass scale from $500 \mathrm{GeV}$ to $10 \mathrm{TeV}$ opens a larger viable region, and at the same time raises the scale $\mu_{*}$. Thus, we find a lower bound of $M_{1} \gtrsim 500 \mathrm{GeV}$.

above $T_{\mathrm{sph}}$. To avoid the false phase transition pattern, we fix as a benchmark value a small singlet self-coupling $\lambda_{S}=0.01$. This ensures that the thermal corrections to the singlet mass parameter are much smaller than to the Higgs mass (see, e.g., [61]). For the resulting asymmetry, however, the numerical value of $\lambda_{S}$ is irrelevant.

With a complete understanding of the relevant bounds and an estimate for the maximal value of $\mu$, chosen such as to limit the washout by scattering processes, we can use the semianalytical estimate, Eq. (22), to efficiently scan the parameter space and identify viable regions. Including the above bounds, we obtain Fig. 6, where we fixed the mass splitting to $\delta_{M}=0.3$. This modest degeneracy is accepted in order to achieve an optimal interplay between large $C P$ asymmetry and not too large washout. This allows us to identify the lowest RHN mass with successful leptogenesis; demanding less degeneracy implies larger RHN masses. The light blue region is excluded because there we obtain an insufficient baryon asymmetry $\left|\eta_{B}\right|<6 \cdot 10^{-10}$, whose sign can always be adjusted, e.g., via the sign of the CI rotation angle. The vertical gray line limits the scalar mass, because we need $m_{S}<M_{2}-M_{1}$ for $\varepsilon_{2}^{\mathrm{v} / \mathrm{s}} \neq 0$. For RHN masses $M_{1} \lesssim 500 \mathrm{GeV}$, this pushes the region with sufficient $C P$ violation beyond the bounds of the scalar singlet model, most importantly the light gray area where $v_{S}=0$, cf. the upper left panel of Fig. 6 . Therefore, $M_{1} \approx 500 \mathrm{GeV}$ is the lowest possible RHN mass which allows for successful baryogenesis, complies 
with all constraints that we considered and predicts an accompanying scalar DOF with $m_{S} \simeq 100 \mathrm{GeV}$. Raising the RHN mass scale to, say, $M_{1}=10 \mathrm{TeV}$, a larger viable region opens up (right panels of Fig. 6). Since at the same time $\mu_{*}$ grows, we find that the accompanying scalar can have a mass in the range $300 \mathrm{GeV} \lesssim m_{S} \lesssim 3000 \mathrm{GeV}$. Remarkably enough, the viable parameter region in this case can be probed by searches for resonant scalar-singlet production at current and future colliders (see, e.g., the shaded region labeled with "CMS $13 \mathrm{TeV}$ ").

\section{CONCLUSIONS AND OUTLOOK}

Baryogenesis via leptogenesis is an appealing mechanism that connects the phenomenology of neutrino oscillations at low energies to the generation of the BAU. Standard thermal leptogenesis in the type-I seesaw model, however, requires very heavy right-handed neutrinos, making it hard to probe this scenario experimentally and resulting in large radiative corrections to the Higgs mass. Currently, the most popular approaches for successful leptogenesis with lower RHN masses rely either on resonant enhancement due to a highly degenerate RHN spectrum or on RHN flavor oscillations.

In this paper, we explored a particularly simple modification of the type-I seesaw framework that enhances $C P$ violation and the departure from thermal equilibrium such that the mass of the introduced RHNs can be accessed in Earth-based experiments. This modification relies only on two RHNs and one new singlet scalar and represents the low-energy effective theory of a large class of possible UV-complete models. Coupling the singlet scalar, $S$, to the RHNs, $N_{1,2}$, opens up a new $N_{2}$ decay channel, $N_{2} \rightarrow N_{1} S$, leading to the above mentioned enhancement of $C P$ violation and departure from thermal equilibrium during the $N_{2}$ decays. Together, these two effects increase the efficiency of leptogenesis, such that the BAU can be successfully generated in $N_{2}$ decays for RHN masses even below the $\mathrm{TeV}$ scale and without the need for a highly degenerate RHN mass spectrum.

We derived a semianalytical fit function for the final baryon asymmetry that allows for an efficient study of the parameter space, while reproducing the exact numeri- cal result with high precision. Using the semianalytical fit, we were able to determine the dependence of the final asymmetry on the choice of parameter values in the neutrino sector taking into account the the most recent low-energy data on neutrino oscillations. We showed that the requirement of successful leptogenesis singles out an interesting region in parameter space that can be probed in on-going and future experiments. In the present analysis, we were able to find viable scenarios with the lightest RHN mass down to roughly $500 \mathrm{GeV}$, but this bound should merely be regarded as a rough estimate. We leave the refinement our analysis that would allow for determining a more precise lower bound on $M_{1}$ for future work. Most importantly, Eq. (21) should be generalized to take into account flavor effects, which we disregarded in the present analysis in order to focus on the new kinematic enhancement due to the singlet scalar. Furthermore, given that the lowest masses that we found lie around the EW scale, a more detailed treatment of sphaleron freeze-out at temperatures around the EW phase transition should also be considered.

In addition, it would be interesting to embed our scenario into concrete UV-complete models that would allow to predict some of our model parameters such as the Yukawa matrix, $\alpha$, or the trilinear coupling, $\mu$. However, all of these questions are beyond the scope of this work. We conclude by emphasizing that the extension of the type-I seesaw model by a real scalar singlet has exciting implications for low-scale leptogenesis that call for further investigation.

\section{ACKNOWLEDGMENTS}

The authors would like to thank Alexander Helmboldt for helpful discussions on the real-scalar-singlet extension of the Higgs sector. The authors are also grateful to Filippo Sala for discussions on the CMS searches for resonant singlet production at the $\mathrm{LHC}$ as well as for making available to us some of the numerical data presented in Ref. [71]. M. P. acknowledges funding from the International Max Planck Research School for Precision Tests of Fundamental Symmetries (IMPRS-PTFS). T. H. and M. P. are enrolled at the University of Heidelberg. 


\section{Appendix A: Loop functions}

The loop functions relevant for the $C P$-asymmetry parameters are given by [46]

$$
\begin{gathered}
\mathcal{F}_{i j, R}^{(\mathrm{v})}=\sqrt{r_{j i}} \ln \left[\frac{\left(1+\eta_{i}\right)\left(1-r_{j i}\right)-\left(1-\eta_{i}\right)\left(\sigma_{i}+\sqrt{\delta_{j i}}\right)}{\left(1+\eta_{i}\right)\left(1-r_{j i}\right)-\left(1-\eta_{i}\right)\left(\sigma_{i}-\sqrt{\delta_{j i}}\right)}\right], \\
\mathcal{F}_{i j, L}^{(\mathrm{v})}=-\sqrt{\delta_{j i}}+\frac{r_{j i}-\eta_{i}}{1-\eta_{i}} \ln \left[\frac{\left(1+\eta_{i}\right)\left(1-r_{j i}\right)-\left(1-\eta_{i}\right)\left(\sigma_{i}+\sqrt{\delta_{j i}}\right)}{\left(1+\eta_{i}\right)\left(1-r_{j i}\right)-\left(1-\eta_{i}\right)\left(\sigma_{i}-\sqrt{\delta_{j i}}\right)}\right], \\
\mathcal{F}_{i j k, R R}^{(\mathrm{s})}=\frac{\sqrt{r_{j i}} \sqrt{r_{k i}} \sqrt{\delta_{j i}}}{1-r_{j i}}, \\
\mathcal{F}_{i j k, R L}^{(\mathrm{s})}=\frac{1}{2} \frac{\sqrt{r_{k i}} \sqrt{\delta_{j i}}\left(1+r_{j i}-\sigma_{i}\right)}{1-r_{j i}} \\
\mathcal{F}_{i j k, L L}^{(\mathrm{s})}=\frac{\sqrt{r_{j i}} \sqrt{\delta_{j i}}}{1-r_{j i}}, \\
\mathcal{F}_{i j k, L R}^{(\mathrm{s})}=\frac{1}{2} \frac{\sqrt{\delta_{j i}}\left(1+r_{j i}-\sigma_{i}\right)}{1-r_{j i}},
\end{gathered}
$$

where $r_{i j} \equiv M_{i}^{2} / M_{j}^{2}, \sigma_{i} \equiv m_{S}^{2} / M_{i}^{2}, \eta_{i} \equiv m_{h}^{2} / M_{i}^{2}$ and $\delta_{i j} \equiv\left(1-r_{i j}-\sigma_{j}\right)^{2}-4 r_{i j} \sigma_{j}$. Note that during leptogenesis (before the EW phase transition), the zero-temperature Higgs boson mass vanishes, $m_{h}=0$. This is not necessarily the case if a scalar doublet other than the SM Higgs is involved in leptogenesis, like for example in the scotogenic model.

[1] N. Aghanim et al. (Planck), (2018), arXiv:1807.06209 [astro-ph.CO].

[2] M. Tanabashi et al. (Particle Data Group), Phys. Rev. D98, 030001 (2018).

[3] M. Fukugita and T. Yanagida, Phys. Lett. B174, 45 (1986).

[4] P. Minkowski, Phys. Lett. 67B, 421 (1977).

[5] T. Yanagida, Proceedings: Workshop on the Unified Theories and the Baryon Number in the Universe: Tsukuba, Japan, February 13-14, 1979, Conf. Proc. C7902131, 95 (1979).

[6] T. Yanagida, Prog. Theor. Phys. 64, 1103 (1980).

[7] M. Gell-Mann, P. Ramond, and R. Slansky, Supergravity Workshop Stony Brook, New York, September 27-28, 1979, Conf. Proc. C790927, 315 (1979), arXiv:1306.4669 [hepth].

[8] R. N. Mohapatra and G. Senjanovic, Phys. Rev. Lett. 44, 912 (1980).

[9] P. S. B. Dev, P. Di Bari, B. Garbrecht, S. Lavignac, P. Millington, and D. Teresi, Int. J. Mod. Phys. A33, 1842001 (2018), arXiv:1711.02861 [hep-ph].

[10] M. Drewes, B. Garbrecht, P. Hernandez, M. Kekic, J. Lopez-Pavon, J. Racker, N. Rius, J. Salvado, and D. Teresi, Int. J. Mod. Phys. A33, 1842002 (2018), arXiv:1711.02862 [hep-ph].

[11] B. Dev, M. Garny, J. Klaric, P. Millington, and D. Teresi, Int. J. Mod. Phys. A33, 1842003 (2018), arXiv:1711.02863 [hep-ph].

[12] S. Biondini et al., Int. J. Mod. Phys. A33, 1842004 (2018), arXiv:1711.02864 [hep-ph].

[13] E. J. Chun et al., Int. J. Mod. Phys. A33, 1842005 (2018), arXiv:1711.02865 [hep-ph].

[14] C. Hagedorn, R. N. Mohapatra, E. Molinaro, C. C. Nishi, and S. T. Petcov, Int. J. Mod. Phys. A33, 1842006 (2018), arXiv:1711.02866 [hep-ph].
[15] G. Bambhaniya, P. Bhupal Dev, S. Goswami, S. Khan, and W. Rodejohann, Phys. Rev. D95, 095016 (2017), arXiv:1611.03827 [hep-ph].

[16] T. Rink and K. Schmitz, JHEP 03, 158 (2017), arXiv:1611.05857 [hep-ph].

[17] T. Rink, K. Schmitz, and T. T. Yanagida, (2016), arXiv:1612.08878 [hep-ph].

[18] V. A. Kuzmin, V. A. Rubakov, and M. E. Shaposhnikov, Phys. Lett. 155B, 36 (1985).

[19] S. Davidson and A. Ibarra, Phys. Lett. B535, 25 (2002), arXiv:hep-ph/0202239 [hep-ph].

[20] W. Buchmuller, P. Di Bari, and M. Plumacher, Nucl. Phys. B643, 367 (2002), [Erratum: Nucl. Phys. B793, 362 (2008)], arXiv:hep-ph/0205349 [hep-ph].

[21] G. F. Giudice, A. Notari, M. Raidal, A. Riotto, and A. Strumia, Nucl. Phys. B685, 89 (2004), arXiv:hepph/0310123 [hep-ph].

[22] W. Buchmuller, P. Di Bari, and M. Plumacher, Annals Phys. 315, 305 (2005), arXiv:hep-ph/0401240 [hep-ph].

[23] T. Hambye, Y. Lin, A. Notari, M. Papucci, and A. Strumia, Nucl. Phys. B695, 169 (2004), arXiv:hep-ph/0312203 [hep-ph].

[24] S. Blanchet and P. Di Bari, Nucl. Phys. B807, 155 (2009), arXiv:0807.0743 [hep-ph].

[25] S. Antusch, S. Blanchet, M. Blennow, and E. FernandezMartinez, JHEP 01, 017 (2010), arXiv:0910.5957 [hep-ph].

[26] K. Moffat, S. Pascoli, S. T. Petcov, H. Schulz, and J. Turner, Phys. Rev. D98, 015036 (2018), arXiv:1804.05066 [hep-ph].

[27] F. Vissani, Phys. Rev. D57, 7027 (1998), arXiv:hepph/9709409 [hep-ph].

[28] I. Brivio and M. Trott, Phys. Rev. Lett. 119, 141801 (2017), arXiv:1703.10924 [hep-ph].

[29] V. Brdar, Y. Emonds, A. J. Helmboldt, and M. Lindner, (2018), arXiv:1807.11490 [hep-ph]. 
[30] I. Brivio and M. Trott, (2018), arXiv:1809.03450 [hep-ph].

[31] V. Brdar, A. J. Helmboldt, and J. Kubo, (2018), arXiv:1810.12306 [hep-ph].

[32] A. Pilaftsis, Phys. Rev. D56, 5431 (1997), arXiv:hepph/9707235 [hep-ph].

[33] A. Pilaftsis and T. E. J. Underwood, Nucl. Phys. B692, 303 (2004), arXiv:hep-ph/0309342 [hep-ph].

[34] E. K. Akhmedov, V. A. Rubakov, and A. Yu. Smirnov, Phys. Rev. Lett. 81, 1359 (1998), arXiv:hep-ph/9803255 [hep-ph].

[35] A. Abada, G. Arcadi, V. Domcke, M. Drewes, J. Klaric, and M. Lucente, (2018), arXiv:1810.12463 [hep-ph].

[36] J. Ghiglieri and M. Laine, (2018), arXiv:1811.01971 [hep$\mathrm{ph}$.

[37] D. Aristizabal Sierra, M. Losada, and E. Nardi, Phys. Lett. B659, 328 (2008), arXiv:0705.1489 [hep-ph].

[38] E. Ma, Phys. Rev. D73, 077301 (2006), arXiv:hepph/0601225 [hep-ph].

[39] T. Hugle, M. Platscher, and K. Schmitz, Phys. Rev. D98, 023020 (2018), arXiv:1804.09660 [hep-ph].

[40] D. Borah, A. Dasgupta, and S. K. Kang, (2018), arXiv:1806.04689 [hep-ph].

[41] S. Baumholzer, V. Brdar, and P. Schwaller, JHEP 08, 067 (2018), arXiv:1806.06864 [hep-ph].

[42] W.-C. Huang, H. Päs, and S. Zeißner, Phys. Rev. D98, 075024 (2018), arXiv:1806.08204 [hep-ph].

[43] D. Borah, P. S. B. Dev, and A. Kumar, (2018), arXiv:1810.03645 [hep-ph].

[44] D. Borah, A. Dasgupta, and S. K. Kang, in 39th International Conference on High Energy Physics (ICHEP 2018) Seoul, Gangnam-Gu, Korea, Republic of, July 4-11, 2018 (2018) arXiv:1811.02094 [hep-ph].

[45] D. Aristizabal Sierra, M. Tortola, J. W. F. Valle, and A. Vicente, JCAP 1407, 052 (2014), arXiv:1405.4706 [hep-ph].

[46] M. Le Dall and A. Ritz, Phys. Rev. D90, 096002 (2014), arXiv:1408.2498 [hep-ph].

[47] V. Silveira and A. Zee, Phys. Lett. 161B, 136 (1985).

[48] J. McDonald, Phys. Rev. D50, 3637 (1994), arXiv:hepph/0702143 [HEP-PH].

[49] C. P. Burgess, M. Pospelov, and T. ter Veldhuis, Nucl. Phys. B619, 709 (2001), arXiv:hep-ph/0011335 [hep-ph].

[50] P. Athron et al. (GAMBIT), Eur. Phys. J. C77, 568 (2017), arXiv:1705.07931 [hep-ph].

[51] R. N. Lerner and J. McDonald, Phys. Rev. D80, 123507 (2009), arXiv:0909.0520 [hep-ph].

[52] F. Kahlhoefer and J. McDonald, JCAP 1511, 015 (2015), arXiv:1507.03600 [astro-ph.CO].

[53] T. Tenkanen, K. Tuominen, and V. Vaskonen, JCAP 1609, 037 (2016), arXiv:1606.06063 [hep-ph].

[54] Y. Ema, M. Karciauskas, O. Lebedev, S. Rusak, and M. Zatta, (2017), arXiv:1711.10554 [hep-ph].

[55] K. Enqvist, S. Nurmi, T. Tenkanen, and K. Tuominen, JCAP 1408, 035 (2014), arXiv:1407.0659 [astro-ph.CO].

[56] J. McDonald, Phys. Lett. B323, 339 (1994).

[57] S. Profumo, M. J. Ramsey-Musolf, and G. Shaughnessy, JHEP 08, 010 (2007), arXiv:0705.2425 [hep-ph].
[58] V. Barger, P. Langacker, M. McCaskey, M. RamseyMusolf, and G. Shaughnessy, Phys. Rev. D79, 015018 (2009), arXiv:0811.0393 [hep-ph].

[59] J. R. Espinosa, T. Konstandin, and F. Riva, Nucl. Phys. B854, 592 (2012), arXiv:1107.5441 [hep-ph].

[60] J. M. Cline and K. Kainulainen, JCAP 1301, 012 (2013), arXiv:1210.4196 [hep-ph].

[61] T. Alanne, K. Tuominen, and V. Vaskonen, Nucl. Phys. B889, 692 (2014), arXiv:1407.0688 [hep-ph].

[62] T. Alanne, A. Meroni, and K. Tuominen, Phys. Rev. D96, 095015 (2017), arXiv:1706.10128 [hep-ph].

[63] O. Lebedev, Eur. Phys. J. C72, 2058 (2012), arXiv:1203.0156 [hep-ph].

[64] J. Elias-Miro, J. R. Espinosa, G. F. Giudice, H. M. Lee, and A. Strumia, JHEP 06, 031 (2012), arXiv:1203.0237 [hep-ph].

[65] V. Barger, P. Langacker, M. McCaskey, M. J. RamseyMusolf, and G. Shaughnessy, Phys. Rev. D77, 035005 (2008), arXiv:0706.4311 [hep-ph].

[66] T. Robens and T. Stefaniak, Eur. Phys. J. C75, 104 (2015), arXiv:1501.02234 [hep-ph].

[67] V. Martín Lozano, J. M. Moreno, and C. B. Park, JHEP 08, 004 (2015), arXiv:1501.03799 [hep-ph].

[68] A. Falkowski, C. Gross, and O. Lebedev, JHEP 05, 057 (2015), arXiv:1502.01361 [hep-ph].

[69] D. Buttazzo, F. Sala, and A. Tesi, JHEP 11, 158 (2015), arXiv:1505.05488 [hep-ph].

[70] J. M. No and M. Spannowsky, (2018), arXiv:1807.04284 [hep-ph].

[71] D. Buttazzo, D. Redigolo, F. Sala, and A. Tesi, JHEP 11, 144 (2018), arXiv:1807.04743 [hep-ph].

[72] P. Di Bari, Nucl. Phys. B727, 318 (2005), arXiv:hepph/0502082 [hep-ph].

[73] J. Casas and A. Ibarra, Nucl.Phys. B618, 171 (2001), arXiv:hep-ph/0103065 [hep-ph].

[74] G. 't Hooft, Recent Developments in Gauge Theories. Proceedings, Nato Advanced Study Institute, Cargese, France, August 26 - September 8, 1979, NATO Sci. Ser. B 59, 135 (1980).

[75] B. Pontecorvo, Sov. Phys. JETP 7, 172 (1958), [Zh. Eksp. Teor. Fiz.34,247(1957)].

[76] Z. Maki, M. Nakagawa, and S. Sakata, Prog. Theor. Phys. 28, 870 (1962), [,34(1962)].

[77] I. Esteban, M. C. Gonzalez-Garcia, A. HernandezCabezudo, M. Maltoni, and T. Schwetz, (2018), arXiv:1811.05487 [hep-ph].

[78] L. Covi, E. Roulet, and F. Vissani, Phys. Lett. B384, 169 (1996), arXiv:hep-ph/9605319 [hep-ph].

[79] M. D'Onofrio, K. Rummukainen, and A. Tranberg, Phys. Rev. Lett. 113, 141602 (2014), arXiv:1404.3565 [hep-ph].

[80] S. Blanchet and P. Di Bari, JCAP 0606, 023 (2006), arXiv:hep-ph/0603107 [hep-ph].

[81] M. D. Goodsell and F. Staub, Eur. Phys. J. C78, 649 (2018), arXiv:1805.07306 [hep-ph].

[82] A. Ilnicka, T. Robens, and T. Stefaniak, Mod. Phys. Lett. A33, 1830007 (2018), arXiv:1803.03594 [hep-ph]. 
[83] A. M. Sirunyan et al. (CMS), JHEP 06, 127 (2018), arXiv:1804.01939 [hep-ex].

[84] G. Aad et al. (ATLAS), JHEP 11, 206 (2015), arXiv:1509.00672 [hep-ex].

[85] V. Khachatryan et al. (CMS), JHEP 02, 135 (2017), arXiv:1610.09218 [hep-ex].
[86] S. Dittmaier et al. (LHC Higgs Cross Section Working Group), (2011), 10.5170/CERN-2011-002, arXiv:1101.0593 [hep-ph]. 